\title{
A new anisotropic continuum traffic flow model with anticipation driving behavior
}

\author{
Yirong Kang, a Shuhong Yang, b \\ ${ }^{1}$ School of Transportation Engineering, Guizhou Institute of Technology, Guiyang, 550003, China \\ ${ }^{2}$ School of Computer Science and Communication Engineering, Guangxi University of Science and Technology, Liuzhou, 545006, China
}

\begin{abstract}
Based on the anticipation driving car-following model, a new macro traffic flow model is established in this paper by considering the relationship between micro and macro variables. Therefore, the evolution law of traffic flow with anticipation driving effect can be studied from macroscopic level. By using approaches of linear stability analysis, the linear stability discriminant condition of the new macro model to keep the traffic flow stable against small disturbance is obtained. Numerical experiments verify that the model can not only simulate the unique shock wave, rarefaction wave mutation and the dynamic propagation process of small disturbance, but also improve the stability of traffic flow by introducing the information of anticipation driving behavior.
\end{abstract}

\section{INTRODUCTION}

Many traffic models ${ }^{[1-5]}$ have been proposed to study the dynamic evolution process of traffic flow up to date. Complex phenomena such as stop-and-go, traffic waves and small disturbance propagation in actual traffic can be effectively presented in these models. However, the variables in these models are usually dependent on current information and involve little information about drivers' predictions of future traffic conditions. In recent years, the research on driver anticipation effect has attracted great attention from scholars, because it is a common inherent psychological phenomenon among drivers in manual driving mode. Tang et al. ${ }^{[6]}$ discussed from the micro level how the driver's forecast effect (DFE) affects the stability of the car following process and the evolution process of traffic congestion. Zheng et al. ${ }^{[7]}$ introduced expected driving behavior factors and the length of predictions time into the car following model, and investigated the impact of expectation effect on traffic flow through linear stability analysis and nonlinear analysis. In 2019, Wang et al. ${ }^{[8]}$ proposed a new car following model by considering the influence of the trend of headway variation, and the results showed that considering the trend of headway variation could improve the stability of traffic flow. By considering the impact of anticipation traffic trends on traffic flow, Zhang et al. ${ }^{[9]}$ tested the prediction effect of drivers in micro traffic model. Although many forms of anticipation effects have been considered in the car following model of stable traffic flow systems, so far there have been few studies on anticipation driving behavior at the macro scale level ${ }^{[10]}$.

Based on the above reasons, starting from the

ae-mail: kyr6700256@126.com

be-mail: caochao_gxust@qq.com

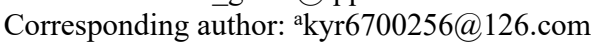

anticipation driving cat following model in literature ${ }^{[7]}$, this paper constructs a new macro traffic flow model with driver anticipation effect through the relationship between micro variables and macro variables of traffic flow. The theoretical analysis and numerical simulation results show that by considering the new factors, the traffic flow can be stabilized on a macroscopic scale and the abrupt phenomena such as shock wave and rarefaction wave observed in traffic can be reproduced.

\section{THE CAR FOLLOWING MODEL}

The car-following model focuses on illustrating the interaction between the adjacent cars on a single lane. The motion equation of traffic flow can be described by the following abstract stimulus response framework ${ }^{[1]}$ :

$$
\frac{d v_{j}(t)}{d t}=g\left(v_{j}, \Delta x_{j}, \Delta v_{j}\right)
$$

In which $v_{j}$ and $x_{j}$ are the speed and location of $j t h$ vehicle respectively. $\Delta v_{j}=v_{j+1}-v_{j}$ and $\Delta x_{j}=x_{j+1}-x_{j}$ denote the relative speed and space headway between the current vehicle and its direct follower. $g(\cdot)$ is a stimulus function that simulates the driver's reaction behavior, and its change depends on the comprehensive effect of the traffic flow speed $v_{j}$, relative speed $\Delta v_{j}$ and headway $\Delta x_{j}$. Later, in order to extend the stimulus response framework to the application scenarios of intelligent transportation system (ITS), scholars introduced different factors and proposed a series of extended car-following models under scenario of ITS.

Considering the headway information of multiple 
vehicles in the intelligent transportation scenario, Ge et al. ${ }^{[11]}$ proposed a cooperative driving model:

$$
\frac{d v_{j}(t)}{d t}=g\left(v_{j}, \Delta x_{j}, \Delta x_{j+1}, \ldots, \Delta x_{j+n}\right)
$$

In 2006, Wang et al. [12] introduced the relative velocity information of multiple leading vehicles into the stimulus response framework and proposed a multi-velocity difference model, i.e

$$
\frac{d v_{j}(t)}{d t}=g\left(v_{j}, \Delta x_{j}, \Delta v_{j}, \Delta v_{j+1} \ldots, \Delta v_{j+k}\right)
$$

In order to further improve the stability of traffic flow, Peng et al. ${ }^{[13]}$ proposed a multi-vehicle following (MCF) model considering multiple speed differences and multiple headway information simultaneously, i.e

$$
\begin{aligned}
& \frac{d v_{j}(t)}{d t}=g\left(v_{j}, \Delta x_{j}, \Delta x_{j+1}, \ldots, \Delta x_{j+p},\right. \\
& \left.\Delta v_{j}, \Delta v_{j+1} \ldots, \Delta v_{j+p}\right)
\end{aligned}
$$

The stability analysis and numerical simulation results of the three models (CD, MVDAND MCF) show that with the help of the intelligent transportation system, the stability of the car following process can be greatly improved and the traffic congestion can be effectively suppressed by the driver's consideration of traffic information in a wider range.

The aforementioned models can describe some complex traffic phenomena, but they cannot be directly used to describe changes in the nature of traffic flow caused by drivers' forecast behavior, because this factor has not been significantly considered. In actual traffic, drivers always adjust the speed of their vehicles dynamically according to the expected dynamic estimation information. Therefore, Zheng et al. ${ }^{[7]}$ proposed an AD-CF model based on the characteristics of anticipation driving behavior:

$$
\frac{d v_{j}(t)}{d t}=k\left\{V\left[\Delta x_{j}(t)+T \Delta v_{j}(t)\right]-v_{j}(t)\right\}+\lambda \Delta v_{j}(t)
$$

where, $T$ is the predicted time, $T \Delta v_{j}(t)$ represents the estimation of the space headway between the leading vehicle and the following one at the next moment. $k$ is the stimulus response coefficient. The idea of the AD-CF model is that the acceleration of the $j t h$ car at time $t$ is not only determined by the speed and the speed difference, but also by the estimated space headway. Therefore, this model can be used to explore the effect of expectation driving behavior on traffic flow in the process of car following on a single lane. When $\mathrm{T}=0$, the $\mathrm{AD}-\mathrm{CF}$ model is reduced to the full velocity difference (FVD) model [5].

For simplicity, $V\left[\Delta x_{j}(t)+T \Delta v_{j}(t)\right]$ in Eq. (5) is linearly expanded employing Taylor expansion method, by ignoring the nonlinear terms, and the following equation can be obtained:

$$
V\left[\Delta x_{j}(t)+T \Delta v_{j}(t)\right]=V\left(\Delta x_{j}(t)\right)+\frac{1}{2} T V^{\prime}\left(\Delta x_{j}(t)\right) \Delta v_{j}(t)
$$

$$
\begin{aligned}
& \frac{d v_{j}(t)}{d t}=k\left[V\left(\Delta x_{j}(t)\right)\right. \\
& \left.+\frac{1}{2} T V^{\prime}\left(\Delta x_{j}(t)\right) \Delta v_{j}(t)-v_{j}(t)\right]+\lambda \Delta v_{j}(t)
\end{aligned}
$$

\section{THE MACRO MODEL WITH ANTICIPATION DRIVING EFFECT}

In order to derive the macro-traffic flow model of driver prediction effect, we converted the micro-variables in Eq. (7) into the corresponding macro-variables based on the transformation method proposed by Liu et al. ${ }^{[14]}$.i.e,

$$
\begin{gathered}
v_{j}(t) \rightarrow v(x, t), v_{j+1}(t) \rightarrow v(x+\Delta, t), \\
V\left(\Delta x_{j}\right) \rightarrow v_{e}(\rho(x, t)), V^{\prime}\left(\Delta x_{j}\right) \rightarrow u_{e}^{\prime}(h)
\end{gathered}
$$

and

$$
k \rightarrow \frac{1}{\eta}, \quad \lambda \rightarrow \frac{1}{\tau}
$$

In which, $v(x, t), \rho(x, t)$, are the velocity and density of point $(x, t)$ respectively; $\Delta$ is the distance between the following and the leading vehicle; $v_{e}(\rho), u_{e}(h)$ represent the equilibrium velocity, and they are defined by density $\rho$ and mean time headway $h=1 / \rho$, respectively. which satisfy $v_{e}(\rho)=u_{e}(h) ; \eta$ is the relaxation time and $\tau$ is the time required for the backward propagating disturbance to propagate a distance $\Delta$. Therefore, Eq. (7) can be rewritten as:

$$
\begin{aligned}
& \frac{\partial v}{\partial t}+v \frac{\partial v}{\partial x}=\frac{v_{e}-v}{\eta}+T u_{e}^{\prime} \frac{v(x+\Delta, t)-v(x, t)}{2 \eta} \\
& +\frac{v(x+\Delta, t)-v(x, t)}{\tau}
\end{aligned}
$$

Expanding Eq. (9) and ignoring the nonlinear terms and combining with the conservation equation of traffic flow, a new macro model considering the impact of anticipation driving behavior can be obtained:

$$
\left\{\begin{array}{c}
\frac{\partial \rho}{\partial t}+v \frac{\partial \rho}{\partial x}+\rho \frac{\partial v}{\partial x}=0 \\
\frac{\partial v}{\partial t}+\left[v-\left(\frac{f u_{e}^{\prime}}{2 \eta}+1\right) c_{0}\right] \frac{\partial v}{\partial x}=\frac{v_{e}-v}{\eta}
\end{array}\right.
$$

Where set $f=T \tau$, and $c_{0}=\frac{\Delta}{\tau}$ represents the propagation speed of small disturbance in traffic flow. When $T=0$ (i.e. $f=0$ ), the driver's expectation behavior is not considered, and the new model degenerates into a speed gradient (SG) model ${ }^{[15]}$.

In order to derive the characteristic velocity of the new model under the driver's expectation behavior, we rewrote Eq. (10) in vector form as follows:

$$
\frac{\partial U}{\partial t}+A \frac{\partial U}{\partial x}=E
$$

Thus, Eq. (5) can be rewritten as follows: 
in which

$$
U=\left[\begin{array}{l}
\rho \\
v
\end{array}\right], \quad E=\left[\begin{array}{c}
0 \\
\frac{v_{e}-v}{\eta}
\end{array}\right], \quad A=\left[\begin{array}{cc}
v & \rho \\
0 & v-\left(u_{e}^{\prime} f / 2 \eta+1\right) c_{0}
\end{array}\right]
$$

Then the eigenvalues $\lambda$ of the matrix $A$ can be obtained by solve eigenproblem as

$$
\operatorname{det}(A-\lambda I)=0
$$

In which $I$ is identity matrix. From equation (12), we get:

$$
\lambda_{1}=v, \quad \lambda_{2}=v-\left(f u_{e}^{\prime} / 2 \eta+1\right) c_{0}
$$

As the equilibrium velocity $u_{e}^{\prime}$ increases with the increase of the headway among vehicles, the characteristic velocity in Equation (13) is not greater than the macro-traffic flow velocity $v$, which illustrate that the model in this paper can well satisfy the basic characteristics of anisotropy of traffic flow ${ }^{[16]}$.

\section{LINEAR STABILITY ANALYSIS}

Suppose $\rho_{0}$ and $v_{0}=v_{e}\left(\rho_{0}\right)$ are steady-state solutions of equation (10).

Small perturbation signal $\xi=\xi(x, t)$ and $\varepsilon=\varepsilon(x, t)$ are introduced into the steady-state solution to obtain the perturbation solution of the new model:

$$
\rho=\rho_{0}+\xi \text { and } v=v_{0}+\varepsilon
$$

Substitute the perturbation solution (14) into Equation (10), then carry out Taylor expansion on $\left(\rho_{0}, v_{0}\right)$, ignore $\xi$ and $\varepsilon$, and get the following expression:

$$
\begin{aligned}
& \xi_{t}+v_{0} \xi_{x}+\rho_{0} \varepsilon_{x}=0 \\
& \varepsilon_{t}+\left[v_{0}-\left(f u_{e}^{\prime} / 2 \eta+1\right) c_{0}\right] \varepsilon_{x}=\frac{v_{e}^{\prime}\left(\rho_{0}\right) \xi-\varepsilon}{\eta}
\end{aligned}
$$

where

$u_{e}^{\prime}=d u_{e} /\left.d h\right|_{h=h_{0}}, \xi_{t}=d \xi / d t, \xi_{x}=d \xi / d x, \varepsilon_{t}=d \varepsilon / d t$, $\varepsilon_{x}=d \varepsilon / d x, v_{e}^{\prime}=d v_{e} / d \rho$.

Eliminating $\varepsilon$ from Eq. (15) and Eq. (16) get the following equation:

$$
\left(\partial_{t}+c \partial_{x}\right) \xi=-\eta\left[\left(\partial_{t}+c_{1} \partial_{x}\right)\left(\partial_{t}+c_{2} \partial_{x}\right)\right] \xi
$$

where $c=v_{e}^{\prime}\left(\rho_{0}\right) \rho_{0}+v_{0}, c_{1}=v_{0}-\left(f u_{e}^{\prime} / 2 \eta+1\right) c_{0}$ and $c_{2}=v_{0}$. Following the traditional way of linear stability analysis, substituting $\xi(x, t)=\xi_{0} \exp (i(\gamma x-w t))$ into (17), and we get

$$
(-i w+i c \gamma) \xi=-\eta\left[\left(-i w+c_{1} i \gamma\right)\left(-i w+c_{2} i \gamma\right)\right] \xi
$$

For $\xi$ to be the non-trivial solution of Eq.(18), the following equation must hold,

$$
\eta\left(w-c_{1} \gamma\right)\left(w-c_{2} \gamma\right)+i(w-c \gamma)=0
$$

It is clear that the solutions are stable if and only if the imaginary parts of both roots $w$ of the equation are not positive. Based on the method used in Ref.[15], this requirement can be expressed as

$$
c_{1} \leq c \leq c_{2}
$$

When condition (20) cannot be satisfied, the traffic flow will be unstable, and many complex traffic phenomena will appear in macroscopic traffic flow, such as stop-and-go or traffic jam.

\section{NUMERICAL SIMULATION}

In order to implement numerical simulation, the finite difference method is used to discretize Eq.(10) to obtain the following difference equation:

$$
\rho_{i}^{j+1}=\rho_{i}^{j}+\frac{\Delta t}{\Delta x} \rho_{i}^{j}\left(v_{i}^{j}-v_{i+1}^{j}\right)+\frac{\Delta t}{\Delta x} v_{i}^{j}\left(\rho_{i-1}^{j}-\rho_{i}^{j}\right)
$$

If the traffic is heavy, $v_{i}^{j}<\left(f u_{e}^{\prime} / 2 \eta+1\right) c_{0}$,

$$
v_{i}^{j+1}=v_{i}^{j}+\frac{\Delta t}{\Delta x}\left[\left(f u_{e}^{\prime} / 2 \eta+1\right) c_{0}-v_{i}^{j}\right]\left(v_{i+1}^{j}-v_{i}^{j}\right)+\frac{\Delta t}{\eta}\left(v_{e}-v_{i}^{j}\right)
$$

Otherwise

$v_{i}^{j+1}=v_{i}^{j}+\frac{\Delta t}{\Delta x}\left[\left(f u_{e}^{\prime} / 2 \eta+1\right) c_{0}-v_{i}^{j}\right]\left(v_{i}^{j}-v_{i-1}^{j}\right)+\frac{\Delta t}{\eta}\left(v_{e}-v_{i}^{j}\right)$

Where, $i, j, \Delta x$ and $\Delta t$, are space index, time index, space step and time step respectively.

Usually, we test the basic performance of the model by examining whether the model can capture the shock wave and rarefaction wave of real traffic flow. Follow this basic line. We set the initial conditions as follows $[10,15]$.

$$
\begin{aligned}
& \rho_{u}^{1}=0.04(\mathrm{veh} / \mathrm{m}), \quad \rho_{d}^{1}=0.18(\mathrm{veh} / \mathrm{m}) \\
& \rho_{u}^{2}=0.18(\mathrm{veh} / \mathrm{m}), \quad \rho_{d}^{2}=0.04(\mathrm{veh} / \mathrm{m})
\end{aligned}
$$

where $\rho_{u}$ and $\rho_{d}$ are the upstream density distribution and downstream density distribution of traffic flow respectively.

The shock wave and rarefaction wave characteristics of macro traffic flow are described by conditions (23a) and (23b) respectively, and the initial velocities are:

$$
v_{u}^{1,2}=v_{e}\left(\rho_{u}^{1,2}\right), \quad v_{d}^{1,2}=v_{e}\left(\rho_{d}^{1,2}\right)
$$

In order to compare with the result of Ref. ${ }^{[15]}$, the following equilibrium velocity ${ }^{[17]}$ is used here:

$$
v_{e}(\rho)=v_{f}\left[1-\exp \left(1-\exp \left(\frac{c_{m}}{v_{f}}\left(\frac{\rho_{j}}{\rho}-1\right)\right)\right)\right]
$$

Where $v_{f}$ is the speed of free traffic flow, and $c_{m}$ is the kinematic wave speed at jam density $\rho_{j}$.Therefore, $u_{e}(h)$ can be defined as [10]:

$$
u_{e}(h)=v_{f}\left[1-\exp \left(1-\exp \left(\frac{c_{m}}{v_{f}}\left(h \rho_{j}-1\right)\right)\right)\right]
$$

where $h=1 / \rho$ is the mean headway. Free boundary condition is adopted here, i.e, we set $\partial v / \partial x=0$ and 
$\partial \rho / \partial x=0$ on both sides. The $20 \mathrm{~km}$ test road is divided into 100 sections equally. Other inputting parameters are as follows:

$$
\begin{aligned}
& v_{f}=30 \quad \mathrm{~m} / \mathrm{s} \quad, \quad c_{m}=c_{0}=11 \quad \mathrm{~m} / \mathrm{s} \\
& \eta=10 \mathrm{~s}, \rho_{j}=0.2 \mathrm{veh} / \mathrm{m}, \Delta x=200 \mathrm{~m}, \Delta t=1 \mathrm{~s}, f=3 \\
& s .
\end{aligned}
$$

The calculation results corresponding to the two Riemann initial conditions (23a) and (23b) are shown in Fig. 1(a) and (b) respectively.

Eq.(23a) simulates a scenario in which a free-moving traffic flow encounters a nearly stopped downstream traffic and a shock wave occurs. Eq. (23b) simulates the physical motion process of the gradually dissipating congestion of traffic flow. In this case, rarefaction waves will appear. Fig.1(a) and (b) successfully reproduce the shock wave and rarefaction waves in macro-traffic flow, indicating that our model can provide correct prediction and simulation of the formation and propagation of traffic flow wave under driver expectation effect. In addition, due to the expectation driving behavior effect taken into account in our model, the transition interface between congestion and free flow is smoother than that in Ref. ${ }^{[15]}$, which means that considering the drivers' prediction of the changing trend of traffic flow ahead has a significant smoothing effect on the traffic flow under different moving conditions.

In order to further study the influence of anticipation driving behavior on traffic flow, the evolution law of small disturbance is studied. In this paper, the initial conditions are selected as follows ${ }^{[18]}$ :

$$
\begin{gathered}
\rho(x, 0)=\rho_{0}+\Delta \rho\left\{\cosh ^{-2}\left[\frac{160}{L}\left(x-\frac{5 L}{16}\right)\right]\right. \\
\left.-\frac{1}{4} \cosh ^{-2}\left[\frac{40}{L}\left(x-\frac{11 L}{32}\right)\right]\right\}
\end{gathered}
$$

where $L=32.2 \mathrm{~km}$ is the length of the road. Periodic boundary conditions are adopted here, namely

$$
\rho(L, t)=\rho(0, t), \quad v(L, t)=v(0, t)
$$

To compare with the results of ${ }^{[15]}$, we use the following speeds here

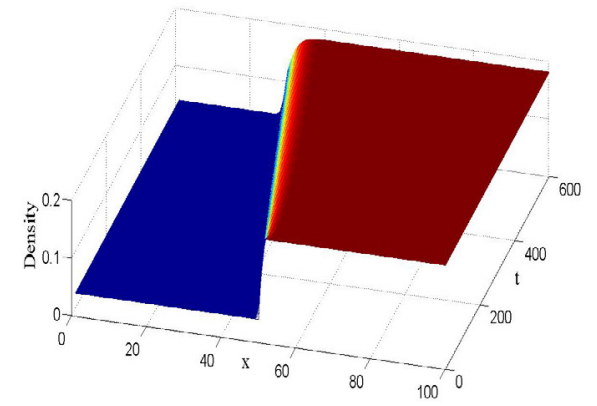

(a)

$$
\begin{aligned}
& v_{e}(\rho) \\
& =v_{f}\left\{\left(1+\exp \left[\frac{\rho / \rho_{\text {jam }}-0.25}{0.06}\right]\right)^{-1}-3.72 \times 10^{-6}\right\}
\end{aligned}
$$

So the equilibrium velocity $u_{e}(h)$ can be defined as $^{[10]}$ :

$$
u_{e}(h)=v_{f}\left\{\left(1+\exp \left[\frac{1 / h \rho_{\text {jam }}-0.25}{0.06}\right]\right)^{-1}-3.72 \times 10^{-6}\right\}
$$

Suppose the initial traffic flow to be in local equilibrium, for example $v(x, 0)=v_{e}(\rho(x, 0))$. we set $\Delta \rho=0.01, \Delta x=100 \mathrm{~m}, \Delta t=1 \mathrm{~s}$ in the simulation below, and other parameters are the same as in Fig. 1. The numerical results at different initial densities are shown in Fig. 2.

The following observations can be obtained from Fig.2.

(i)When $\rho_{0} \leq 0.03$ (traffic flow is in a low-density free flow state) or $\rho_{0} \geq 0.08$ (in a high-density state traffic congestion), the traffic flow behave as we expect, and the small disturbance signals dissipate rapidly rather than propagating and amplifying gradually. Compared with the results of Ref. ${ }^{[15]}$, in this case, the driver's anticipation behavior has little influence on the macro-evolution trend of traffic flow.(See Figure 2(a) and (d))

(ii) Compared with the results of SG model ${ }^{[15]}$, in the density interval $0.03<\rho_{0}<0.08$, the anticipation driving behavior can further improve the motion stability of macro traffic flow, and has a positive effect on restrains small disturbance of traffic flow (see Fig.2(b)).

(iii)When $0.03<\rho_{0}<0.08$, as the initial density $\rho_{0}$ increases, as shown in Fig. 2(b)-(c), the operating efficiency of the traffic system becomes worse, and the macro traffic flow gradually evolves into multiple clusters with uneven distribution under small disturbance, which is of great significance to the stop-and-go working conditions in real world.

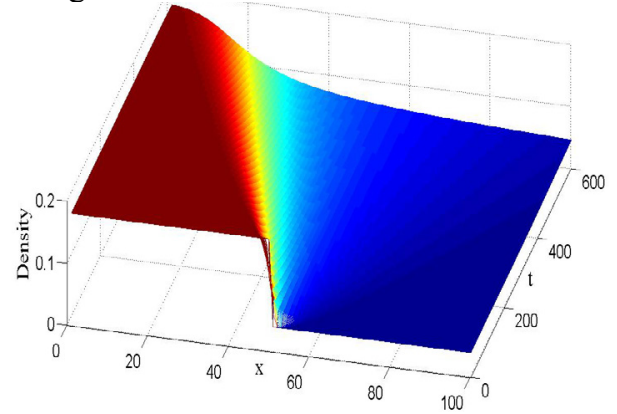

(b)

Fig.1. The density evolution under the initial conditions (23), where (a) shock wave corresponds to Eq.(23a), (b) rarefaction wave corresponds to Eq.(23b). 


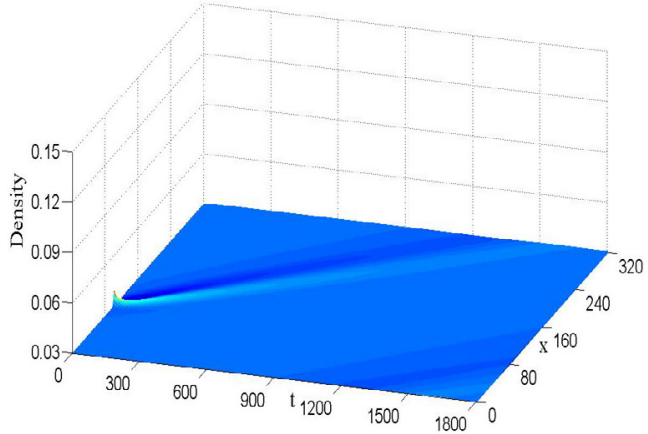

(a)

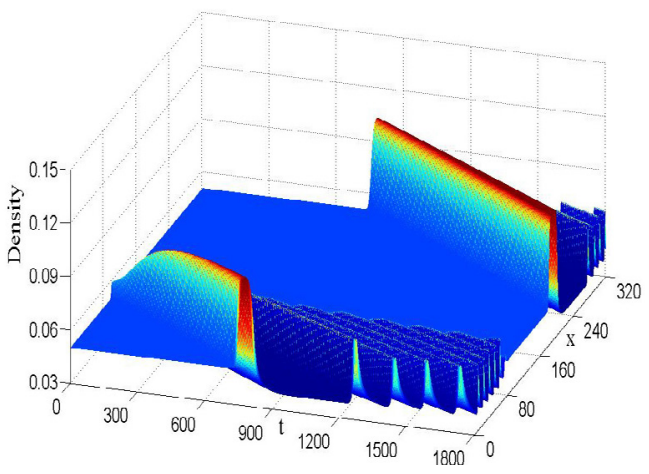

(c)

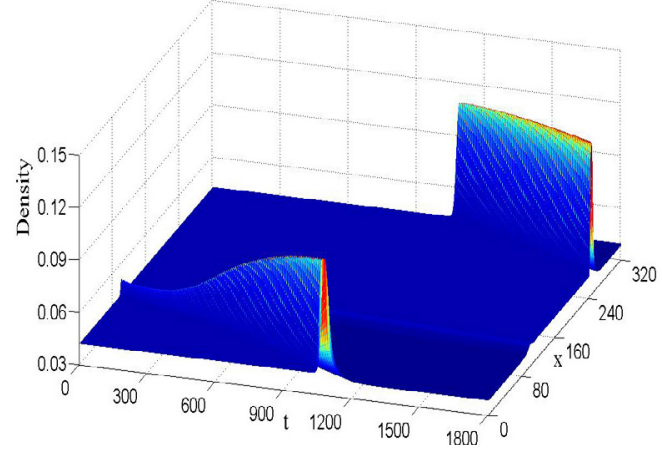

(b)

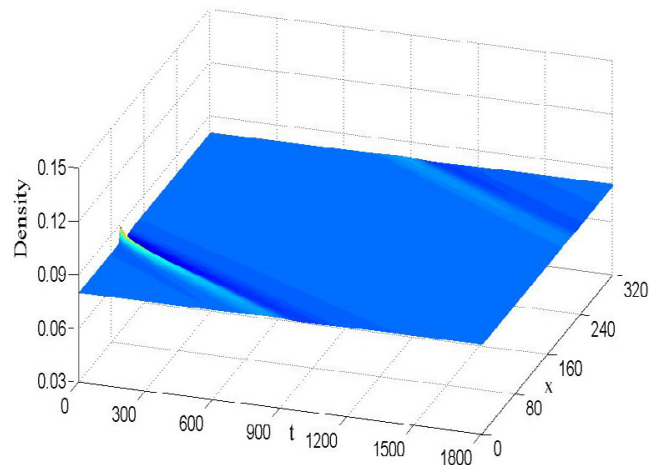

(d)

Fig.2. Temporal evolution of density under the small perturbation for (a) $\rho_{0}=0.03$,(b) $\rho_{0}=0.042$,(c) $\rho_{0}=0.05$ and(d) $\rho_{0}=0.08$, where the units of the $t$-axis, the $x$-axis and the density-axis are second, $100 m$ and $v e h / m$, respectively.

\section{Conclusions}

Starting from a car following model describing the expected driving behavior of actual traffic, and based on the relationship between micro and macro variables in the physical process of traffic movement, a new macro model of traffic flow is established, which lays a foundation for the study of the dynamic evolution characteristics of traffic flow under the driver's anticipation effect. By using the theory of linear stability, the criterion of system stability under the condition of small perturbation is obtained. The results of eigenvalue analysis show that the model satisfies the basic characteristics of anisotropy of traffic flow. The numerical simulation results show that the model can reproduce the complex phenomena such as rarefaction wave, shock wave, stop-go and small disturbance effect in real traffic, and the anticipation driving behavior can improve the stability of traffic flow and smooth the traffic fluctuation.

\section{Conflict of Interests}

The authors declare that there is no conflict of interests regarding the publication of this paper.

\section{Acknowledgements}

This work is supported by Science and Technology Plan Projects of Guizhou Province:(No. [2018]1059), Natural Science Foundation of Guangxi (No.2018GXNSFAA050020) and Science and Technology Plan Projects of Guizhou Province: (No. [2019]1151).

\section{References}

1. Chowdhury D., Santen L., (2000) Schadschneider A. Statistical physics of vehicular traffic and some related systems. Phys Rep, 329:199-29.

2. Kaur, R., Sharma, S. (2017) Analysis of driver's characteristics on a curved road in a lattice model. Physica A, 471:59-67.

3. Peng, G.H., Kuang, H., Qing, L. (2018) Feedback control method in lattice hydrodynamic model under honk environment. Physica A, 509:651-656.

4. Peng, G.H., Yang, S.H., Zhao, H.Z. (2018) New feedback control model in the lattice hydrodynamic model considering the historic optimal velocity difference effect. Commun. Theor. Phys,70:803-807. 
5. Jiang, R., Wu, Q.S., Zhu, Z.J. (2001) Full velocity difference model for a car-following theory. Phys Rev E, 64:017101.

6. Tang, T.Q., Li, C.Y., Huang, H, J. (2010) A new car-following model with the consideration of the driver's forecast effect. Phys lett A, 374: 3951-56.

7. Zheng, L.J., Tian, C., Sun, D.H., Liu, W.N. (2012) A new car-following model with consideration of anticipation driving behavior. Nonlinear Dynam, 70, 1205-1211.

8. Wang, T., Li, G.Y., Zhang, J., Li, S.B., Sun, T. (2019) The effect of Headway Variation Tendency on traffic flow: Modeling and stabilization. Physica A 525, 566-575.

9. Zhang, J., Wang, B., Li, S.B., Sun, T., Wang, T. (2020) Modeling and application analysis of car-following model with predictive headway variation. Physica A 540, 123171.

10. Tang, T.Q., Huang, H.J., Shang, H.Y. (2010) A new macro model for traffic flow with the consideration of the driver's forecast effect. Phys Lett A, 374: 1668-72.

11. Ge, H.X., Dai, S.Q., Dong, L.Y., Xue, Y. (2010) Stabilization effect of traffic flow in an extended car-following model based on an intelligent transportation system application. Phys Rev E, 70: 066134-9.

12. Wang, T., Gao, Z.Y., Zhao, X.M. (2006) Multiple velocity difference model and its stability analysis. Acta Phys Sin, 55: 634-40.

13. Peng, G.H., Sun, D.H. (2010) A dynamical model of car-following with the consideration of the multiple information of preceding cars. Phys lett A, 374: 1694-98.

14. Liu, G.Q., Lyrintzis, A.S., Michalopoulos, P.G. (1998) Improved high-order model for freeway traffic flow. Transp Res Rec, 1644: 37-46.

15. Jiang, R, Wu, Q.S., Zhu, Z.J. (2002) A new continuum model for traffic flow and numerical tests. Transp Res B, 36 :405-19.

16. Daganzo, C.F. (1995) Requiem for second-order fluid approximation of traffic flow. Transp Res B, 29: 277-86.

17. Del Castillo, J.M., Pintado, P., Benitez, F.G. (1994) The reactive time of drivers and the stability of traffic flow. Transp Res B, 28: 35-60.

18. Herrmann, M., Kerner, B.S. (1998) Local cluster effect in different traffic flow models. Physica A, 255:163-88.

19. Kerner, B.S., Konhauser, P. (1993) Cluster effect in initially homogeneous traffic flow. Phys Rev E, 48: R2335-38. 